\title{
Editorial: JFM at 50
}

\author{
By S. H. DA VIS AND T. J. PEDLEY
}

A fiftieth birthday encourages one to look both backwards and forwards. Could the behaviour of the infant, in the first year or two of life, give a glimpse of the adult to come? And can the present state of that adult be used to make predictions for the future? Here we refer not to the existence or eminence of the Journal of Fluid Mechanics, both of which seem to us to be perfectly secure, even with the onset of electronic and open-access publishing, but to its content and the nature of fluid mechanics itself. To give substance to our reflections we have looked through volumes 1 and 2 (May 1956 to August 1957) and compared them with the three most recent volumes to hand at the time of writing, numbers 532 to 534 (June to July 2005). The total number of pages in each case was almost the same, though there were 80 papers in the first two volumes and only 49 in the recent three.

The first thing that strikes one about volumes 1 and 2 is the very high proportion of authors who were, or later became, extremely distinguished:

Batchelor (2 papers), Brooke Benjamin (2), Chester (2), Coles, Favre, Freeman (2 papers each), Michael Glauert (3), Greenspan, Hayes, Joe Keller, Kovasznay, Laufer, Lighthill (4 papers), Malkus, Milne-Thompson, Derek Moore, LonguetHiggins, Morton, Owen Phillips, Ian Proudman (2), Joseph Proudman (probably the only example of father and son publishing separate papers in the same volume of JFM), Rott, Saffman (2), Scorer, Shercliff, Stewart, Townsend (4), Turner, Van Dyke, Whitham (2), Les Woods, Yih, etc. Perhaps the only missing name that one might have expected to see is that of G. I. Taylor, who did not publish a paper in JFM until volume 9.

Not surprisingly, many of these papers from famous authors made pioneering, or at least significant, contributions to their fields. Among the highlights of volume 1 are Saffman \& Turner's discussion 'On the collision of drops in turbulent clouds' (pp. 16-30), which could have been written today (and in fact often is!); Lighthill's 'Drift' (pp. 31-53), analysing how a plane of material particles is distorted by the passage of a solid body through it (neglecting viscosity) and hence how weak vorticity is profoundly modified as it passes such a body, which is one of the bases of rapid distortion theory; Batchelor's 'On steady laminar flow with closed streamlines at large Reynolds number' (pp. 177-190); Coles's 'The law of the wake in the turbulent boundary layer' (pp. 191-226), Benjamin's 'On the flow in channels when rigid obtacles are placed in the stream' (pp. 227-248), which followed on from the earlier paper of Benjamin \& Lighthill (1954) but also included some experimental tests of the theory; Saffman's 'On the rise of small air bubbles in water' (pp. 249-275); and 'On the motion of small spheroidal particles in a viscous liquid' (pp. 540-553); Whitham's 'On the propagation of weak shock waves (pp. 290-318); Chester's 'Supersonic flow past a bluff body with a detached shock' in two parts (pp. 353-365 and 490-496); and Shercliff's 'The flow of conducting fluids in circular pipes under transverse magnetic fields' (pp. 644-666). The most prominent paper in volume 2 is undoubtedly Proudman \& Pearson's 'Expansions at small Reynolds numbers for the flow past a sphere and a circular cylinder' (pp. 237-262) which, with Kaplun \& Lagerstrom (1957), developed the rational method of matched asymptotic expansions for 
low-Reynolds-number flow, but there is also another paper by Whitham, 'A new approach to problems of shock dynamics. Part 1. Two-dimensional problems' (pp. 145-171), Phillips's 'On the generation of waves by turbulent wind' (pp. 417-445) and Benjamin's 'Wave formation in laminar flow down an inclined plane' (pp. 554-574).

The majority of these first papers were theoretical studies - 'applied mathematics' in the British sense - but the goal, as in all JFM papers, was physical understanding; however, there were a reasonable number of experimental papers (16) plus quite a few more designed to aid in the interpretation of experimental findings such as Hall's and Lighthill's analyses of the displacement effect of a Pitot tube (vol. 1, pp. 142-162 and vol. 2, pp. 493-512). Not surprisingly, the only computations were very straightforward by modern standards. From all 80 papers, 26 were on supersonic flows, including 15 on shock waves and 3 on non-simple gases, 17 were on incompressible laminar flows, 14 on turbulence, 7 on water waves, 5 on convection, 4 on drops and bubbles, and very few on rotating fluids, geophysical applications, magnetohydrodynamics (MHD), etc.

Turning now to volumes 532-534, one is struck by a number of differences, apart from the (unwelcome) increase in the average length of the papers and the (welcome) reduction in the number of titles beginning 'On the...'. Now the number of predominantly computational papers is large -21 out of 49 - with the number that are either predominantly theoretical or predominantly experimental being equal, at 14 each. There are still a lot of papers on turbulence (12), 8 on hydrodynamic stability, 6 on drops, bubbles and other phenomena involving surface tension, such as foams, but only a small number on any one of a wide range of other topics both traditional, such as water waves (2), and new, such as granular media (4) or biological applications (2). There was only one on supersonic flow.

Another notable feature of the new papers is the rarity of single-authored papers. This is not just a matter of supervisors putting their names on their students' papers; these days fluid mechanics has become much more collaborative and widespread. Many of the papers from volumes 532-534 are collaborations between different institutions, often in different countries. The number of centres in which fluidmechanics research is performed has increased enormously with a corresponding increase in the number of research workers. The papers in volumes 1 and 2 came from 8 countries, but $44 \%$ of them were from two institutions, Cambridge (17 papers) and Manchester (18 papers) (it was not a coincidence that Batchelor was at Cambridge and Lighthill at Manchester!). Several other institutions were the source of two or three papers in those volumes: Cal Tech, Princeton, Johns Hopkins, New York University, Brown, Lehigh, General Electric in the USA; Imperial College, Bristol, the National Physical Laboratory and the National Institute of Oceanography in the UK. The papers in volumes 532-534, on the other hand, come from 16 different countries, and no institution was represented more than four times (Imperial College), with a few threes: Cal Tech, Cambridge and Marseille. Moreover, while there are already famous names among the authors in these volumes, it seems rather unlikely that such a large proportion of the authors will become as famous as those in volumes 1 and 2 . But the broadening and internationalizing of the field can only be welcomed, and we hope that fluid mechanics will spread even more broadly across the world in future.

We have referred to the unwelcome increase in the average length of papers, but further reflection suggests that a long paper is not necessarily to be condemned if it really does contain detailed technical information coupled to pertinent analysis and full discussion to provide a new depth to the understanding of the topic in question, whether new or traditional. The density of new material is the parameter of interest. 
We highlight as an example the longest paper in volumes 532-534: 'Progressive waves with persistent two-dimensional surface patterns in deep water', by J. L. Hammack, D. M. Henderson \& H. Segur (vol. 532, pp. 1-52). In this paper the authors give a description of the results of a meticulous series of experiments on two-dimensional propagating wave patterns and reveal numerous features that require theoretical understanding. They then outline the derivation of the appropriate weakly nonlinear theory, leading to a single or a pair of coupled nonlinear Schrödinger (NLS) equations, and show how almost (but not quite) all the features of interest can be explained in terms of solutions of the NLS equations or perturbations to them. This is a classic problem, and therefore of interest to many fluid dynamicists, the paper is very clearly written and authoritative and it provides considerable satisfaction to the reader. It is hard to see how this work could have been so well described in a shorter paper.

On the other hand these volumes also contain several (10) short papers, of ten pages or less, accepted under the fast-track scheme. These too are generally excellent, emphasizing the point, which would have needed no emphasis fifty years ago, that significant results do not need great length for their exposition. The two shortest papers are 'Far-field description of the flow produced by a source of both momentum and mass' by V. N. Kurdyumov (vol. 532, pp. 191-198), which shows how the LandauSquire jet is modified when there is a source of mass as well as momentum (this could have been done in the 1960s, but apparently was not), and 'Uncovering the physics of flapping flat plates with artificial evolution' by M. Milano \& M. Gharib (vol. 534, pp. 403-409), which uses a neat evolutionary algorithm to find the flapping kinematics that maximizes the average lift generated (experimentally) by a rectangular plate with given frequency, maximum speed and maximum displacement of the centre of mass, with application to insect flight. These two papers are excellent examples of both traditional and totally new problems and methods. The new problems are often exciting but there are traditional ones which remain pertinent today.

So what about the future? In the 1950s one might have predicted the ongoing challenge of turbulence 50 years hence, or the development of nonlinear over linear studies of acoustics, water waves and stability, or the increasing importance of numerical computation in fluid mechanics (though not the phenomenal increase in computer power). One would also have noted the beginnings of important new areas of application such as geophysical fluid dynamics, magnetohydrodynamics and biological fluid dynamics. But it would probably have been hard to predict the richness that would emerge from some classical fields, such as low-Reynolds-number hydrodynamics (and suspensions), or surface-tension-driven phenomena in thin film flows as well as drops and bubbles. It would also have been hard to predict the virtual demise of gas dynamics and supersonic flow theory.

Totally new areas such as complex fluids, geological fluid dynamics and micro/nano phenomena would have occurred to very few people. New developments in mathematics, such as dynamical systems theory and pattern formation and solitons in integrable systems, arose in a fluid dynamical context and have had far-reaching implications. New measurement techniques such as laser-Doppler anemometry and particle-image velocimetry have transformed the experimental scene.

The progress made over the last 50 years has been astonishing, attracting to the field an enormous array of talented scientists and engineers worldwide. These brilliant people have been attracted because of the beauty of the subject, its visual allure and complexity being extremely appealing. They have been attracted by the vitality of a field in which developments are interactive among analysis, experiment and scientific computation. This interaction keeps the research honest by requiring 
that predictions and observations be understood in physical terms. Papers are not accepted for publication in JFM if they do not seek to provide physical understanding.

All we can be confident of, in looking forward over the next 50 years, is that fluid mechanics will continue to provide exciting opportunities for research by talented and imaginative people. Every new technological advance involving fluid flow leads to new fluid-mechanical problems, which are almost always different from the traditional problems because of the inherent nonlinearity of the subject and the richness of the physical effects that impinge on it. There will be new discoveries in the natural universe which require fluid-dynamic understanding (e.g. currently, the formation of planets from stellar accretion disks). And there will remain traditional problems, not only turbulence, which remain topical because of novel engineering applications: for example, the (inviscid) theory of water waves, a 19th century invention, is still of critical interest to those attempting to extract energy from the oceans, or trying to guarantee the integrity of off-shore structures and ships.

Finally, we revert once more to the comparison between volumes 1 and 2 and volumes 532-534. There is one aspect of the changes that have taken place that is a source of regret: the style of book reviews. In volumes 1 and 2 there were ten reviews, none less than $2 \frac{3}{4}$ pages in length and the longest being $6 \frac{1}{4}$ pages (Lighthill on The Structure of Turbulent Shear Flow by A. A. Townsend; vol. 1, pp. 554-560). The reviewers, all distinguished fluid dynamicists, took the opportunity to write essays on the subject of the book, often with remarkable insights to convey, with sometimes rather brief mention of the book itself! Reviewers were not afraid of being critical: Batchelor wrote a $4 \frac{1}{2}$ page review of a chemical engineering textbook of which the first half explained what the aims of such a book should be and the second half pointed out forcibly in how many ways the book in question fell short of those aims (vol. 2, pp. 204-208). The final paragraph of the review is the ultimate put-down:

"It is of course always possible to learn something from a new book. I myself picked up one little wrinkle, which I have stored away mentally for use on occasions when I wish to impress students with my progressive outlook; it is that when one is establishing the equation of continuity, it is nowadays desirable to exclude the possibility that nuclear reactions are going on in the volume element under consideration."

Apart from that review, however, the most enjoyable one to read was Corrsin's scholarly but humorous 6-page review of Surveys in Mechanics, the 70th-birthday tribute to G. I. Taylor, edited by Batchelor \& Davies (vol. 2, pp. 616-621). In the modern three volumes there were only three books reviewed, none of the reviews was more than a page and a half in length and all were more-or-less positive. Perhaps the enhanced pace of modern scientific life does not leave us enough time for essaywriting, though the reading of such essays is one of the pleasures of JFM-browsing.

\section{REFERENCES}

Benjamin, T. B. \& Lighthill, M. J. 1954 On cnoidal waves and bores. Proc. R. Soc. Lond. A 224, 448-460.

Kaplun, S. \& Lagerstrom P. A. 1957 Asymptotic expansions of Navier-Stokes solutions for small Reynolds numbers. J. Math. Mech, 6, 585-593 